\title{
Vertically Aligned Gold Nanorod Monolayer on Arbitrary Substrates: Self-Assembly and Femtomolar Detection of Food Contaminants
}

\author{
Bo Peng, ${ }^{\dagger}$ Guangyuan Li, ${ }^{\dagger}$ Dehui Li, ${ }^{\dagger}$ Stephanie Dodson, ${ }^{\dagger}$ Qing Zhang, ${ }^{\dagger}$ Jun Zhang, ${ }^{\dagger}$ Yih Hong Lee, ${ }^{\S}$ \\ Hilmi Volkan Demir, ${ }^{+, \neq}$Xing Yi Ling, ${ }^{\S}$ and Qihua Xiong ${ }^{t, \neq, *}$ \\ †Division of Physics and Applied Physics, School of Physical and Mathematical Sciences, Nanyang Technological University, Singapore 637371, ${ }^{\ddagger}$ Division of \\ Microelectronics, School of Electrical and Electronic Engineering, Nanyang Technological University, Singapore 639798, and \$Division of Chemistry and Biological \\ Chemistry, School of Physical and Mathematical Sciences, Nanyang Technological University, Singapore 637371
}

\begin{abstract}
Public attention to the food scandals raises an urgent need to develop effective and reliable methods to detect food contaminants. The current prevailing detections are primarily based upon liquid chromatography, mass spectroscopy, or colorimetric methods, which usually require sophisticated and time-consuming steps or sample preparation. Herein, we develop a facile strategy to assemble the vertically aligned monolayer of $\mathrm{Au}$ nanorods with a nominal $0.8 \mathrm{~nm}$ gap distance and demonstrate their applications in the rapid detection of plasticizers and melamine contamination at femtomolar level by surface-enhanced Raman scattering spectroscopy (SERS). The SERS signals of plasticizers are sensitive down to $0.9 \mathrm{fM}$
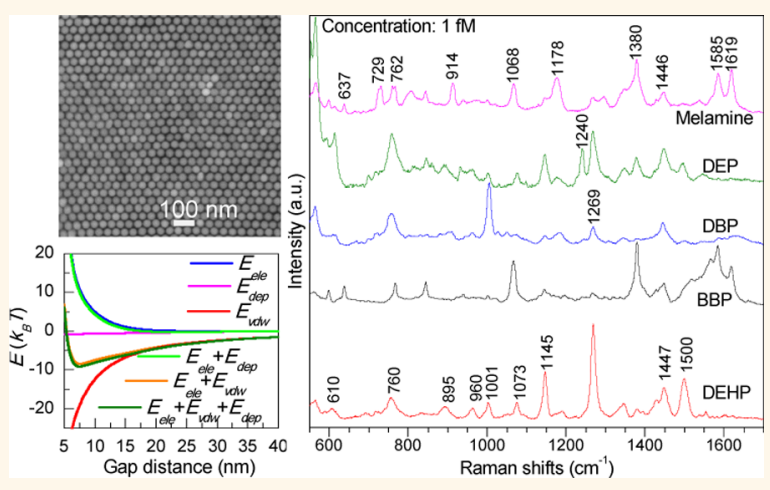
concentrations in orange juices. It is the lowest detection limit reported to date, which is 7 orders of magnitude lower than the standard of United States (6 ppb). The highly organized vertical arrays generate the reproducible "SERS-active sites" and can be achieved on arbitrary substrates, ranging from silicon, gallium nitride, glass to flexible poly(ethylene naphthalate) substrates.
\end{abstract}

KEYWORDS: gold nanorods · self-assembly · vertical monolayer · surface-enhanced Raman scattering · sensors · plasticizers

$\mathrm{F}$ ood and drink safety has become one of the most urgent needs in our daily life, especially since the occurrence of the dioxin egg scandal and recent scandals of melamine in infant formula and plasticizers in food and drinks. ${ }^{1-3}$ Such foodborne hazards come either from environmental hazards (e.g., contamination of phthalate plasticizers from processing equipment such as piping or containers) or from illegal addition driven by the economic benefit (e.g., the melamine in infant formula and plasticizer contaminations in food and drinks). This has imposed a pressing demand for rapid, inexpensive but effective and reliable methods to detect food contamination. However, the current available techniques, such as high-performance liquid chromatography, ${ }^{4}$ mass spectroscopy, ${ }^{5}$ and colorimetric detection, ${ }^{6,7}$ are restricted by time-consuming steps, inadequate detection limits, and complicated sample pretreatment steps such as extraction, preconcentration, and derivatization. An ideal detection strategy should exhibit high sensitivity and specificity and require a minimal sample preparation with rapid detection and low cost. ${ }^{8}$

Recently, the self-assembly of monodisperse metal nanoparticles into ordered arrays has intrigued interest for surface-enhanced Raman scattering (SERS) spectroscopy, which is capable of providing highly resolved specific vibrational molecular information to detect and identify molecules, and requires little sample preparation.9,10 The essential idea toward high sensitivity SERS detection is the engineering of noble metal containing substrates toward a highly localized
* Address correspondence to qihua@ntu.edu.sg.

Received for review April 5, 2013 and accepted June 22, 2013.

Published online June 22, 2013 10.1021/nn401685p

(C) 2013 American Chemical Society 

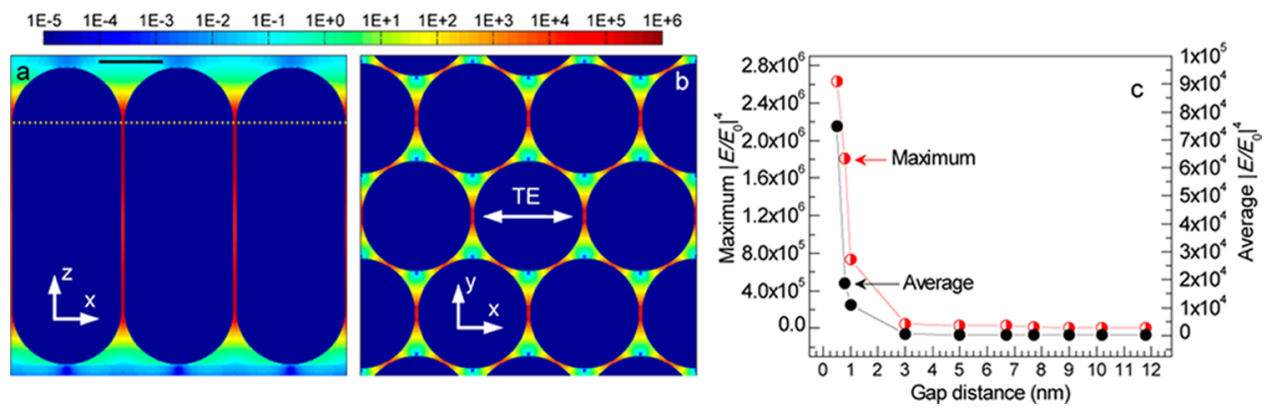

Figure 1. Local electric field simulation. (a,b) Local electric field enhancement factor, $\left|E / E_{0}\right|^{4}$, in the cross section for hexagonal vertical Au nanorod arrays with a $\sim 0.8 \mathrm{~nm}$ edge-to-edge gap distance: (a) along $x-z$ plane, (b) top-view plane along the $y$ axis designated in (a), $x-y$ plane. TE shows the polarization of a $785 \mathrm{~nm}$ laser. (c) Volume maximum and average electric field enhancement factor as a function of the edge-to-edge gap distance.

electromagnetic field. ${ }^{11,12}$ It has been proved that an enhancement factor (EF) value between $1 \times 10^{6}$ and $1 \times 10^{8}$ is adequate to achieve single-molecule detection. ${ }^{13}$ In the past decades, many developments have been achieved on the SERS-active nanostructures, such as Au and Ag nanoparticles, ${ }^{9}$ nanoshells, ${ }^{14}$ and colloidal metal nanoparticle arrays. ${ }^{15}$ However, many SERS-active substrates suffer from poor reproducibility of the "hot spots". ${ }^{16}$ It is thus a major challenge to reproducibly prepare stable SERS substrates with uniform hot spots and possibly push the gaps to the sub-nanometer regime in order to enhance the sensitivity. We hypothesize that a vertical monolayer geometry consisting of $\mathrm{Au}$ nanorods may exhibit highly uniform hot spots. The essential anisotropic shape of Au nanorods also renders the vertical arrays with a strong nanoantenna effect. ${ }^{17,18}$ Finite-difference timedomain method (FDTD) simulation indeed suggests that such a monolayer of hexagonally packed nanorod arrays exhibits strong and uniform local electromagnetic fields. The realistic values for the permittivity of gold as a function of wavelength were used to take the absorption into account. ${ }^{19}$ Figure 1 displays the local electromagnetic field enhancement contour plots in the cross sectional areas of vertical ( $x-z$ plane, Figure 1a) and horizontal ( $x-y$ plane, Figure $1 \mathrm{~b}$ ) planes when the incident plane wave is in transverse electric (TE) polarization. The local electric field enhancement factor is defined as $\left|E / E_{0}\right|^{4}$, where $|E|$ and $\left|E_{0}\right|$ are magnitudes of the local electric field and the incident electric field, respectively. The fields are spatially confined into the gap between adjacent Au nanorods to give a homogeneous and strong near-field localization in 3D spatial structures. The maximum $\left|E / E_{0}\right|^{4}$ values are around $2.6 \times 10^{6}$ and $1.8 \times 10^{6}$ when the gap distance is 0.5 and $0.8 \mathrm{~nm}$, respectively. The volume maximum and average electric field enhancement factors as a function of the edge-to-edge gap distance between two adjacent $\mathrm{Au}$ nanorods are plotted in Figure 1c. $\left|E / E_{0}\right|^{4}$ decreases with an increase of the gap distance, which indicates that gap distance decreases will improve the EF and the sensitivity of SERS. In addition, the gaps between the nanorod "forest" can trap liquid samples due to the capillary force, ${ }^{20-22}$ which is another advantage of the vertical Au nanorod arrays for SERS. Therefore, the highly organized vertical Au nanorod arrays act as an extended nanoantennae to generate a strong, reproducible, and highly homogeneous distribution of electric field, which may provide facile and reproducible SERS substrates that are better than the nanoparticle substrates reported previously. ${ }^{14}$ However, ordered assembly of anisotropic nanorods, particularly in monolayer fashion, is still a major challenge due to more varieties of the assembly modes in nanorods, compared to higher symmetry counterparts, such as nanoparticles. Until recently, only a few successes have been reported on the directional self-assembly of CTAB-stabilized Au nanorods into standing multilayer arrays with a $\sim 3 \mathrm{~nm}$ gap distance. $^{17}$

Herein, we demonstrate a simple but robust approach to self-assemble the CTAB-coated Au nanorods into vertical monolayer arrays with a nominal $0.8 \pm$ $0.3 \mathrm{~nm}$ gap distance. On the basis of the vertically aligned Au nanorod monolayer with sub-nanometer gaps, the food contaminants, plasticizer and melamine, were detected at femtomolar level, 7 orders of magnitude lower than the maximum allowable level of 6 ppb regulated by U.S. Food and Drug Administration (FDA). ${ }^{23}$ Similar sensitivity of $\sim 0.9 \mathrm{fM}$ concentration can be achieved in actual orange juices by intentionally adding trace amount of plasticizers, suggesting great potential toward real applications in food safety screening.

\section{RESULTS AND DISCUSSION}

Self-Assembly of Au Nanorods into the Vertically Aligned Monolayer. We use an evaporation-induced selfassembly strategy to generate the vertically aligned monolayer of CTAB-stabilized Au nanorods based on nearequilibrium status at the internal region of the drying droplet, which eliminates the complex ligand exchange reaction. ${ }^{24-30} \mathrm{Au}$ nanorods with $\sim 92.5 \mathrm{~nm}$ length and $\sim 34.3 \mathrm{~nm}$ diameter were synthesized using CTAB as stabilizer, and their longitudinal plasmon band 

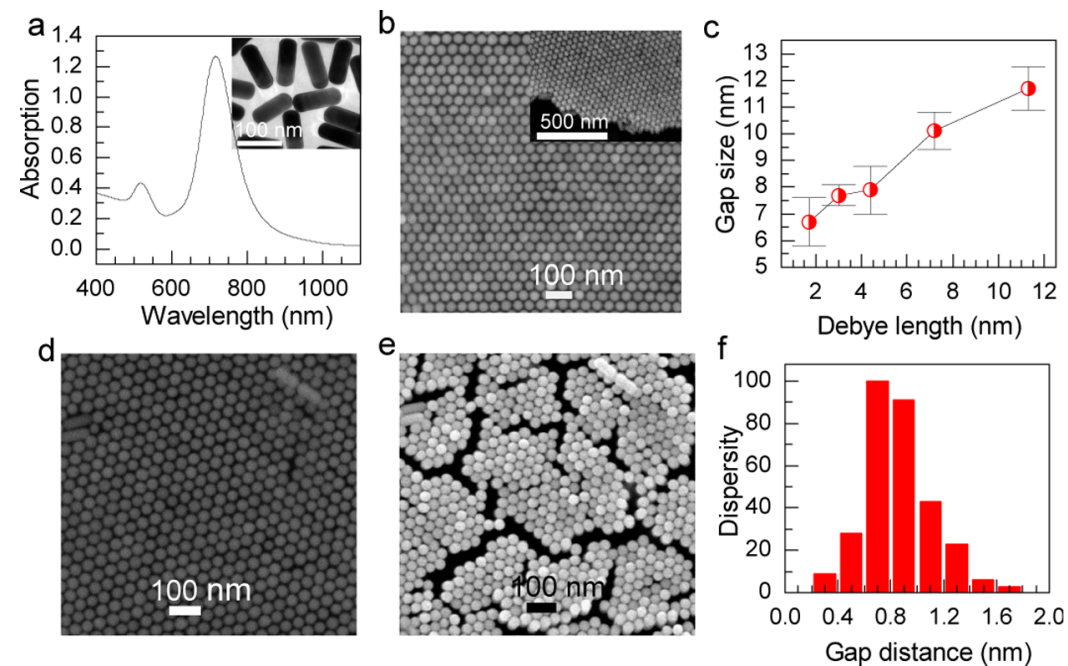

Figure 2. (a) Absorption spectrum of Au nanorod aqueous solution. The inset is the TEM image of Au nanorods. (b) SEM images of vertical Au nanorod monolayer arranged in hexagonal lattice. The edge-to-edge gap distance is $7.7 \pm 0.4 \mathrm{~nm}$; the inset shows the monolayer feature. (c) Measured edge-to-edge gap distance versus the Debye length, adjusted by changing the ionic strength. SEM images of the same vertical Au nanorod arrays before (d) and after (e) treatment by UV ozone. The edge-to-edge gap distance decreases to sub-nanometer, $0.8 \pm 0.3 \mathrm{~nm}$. (f) Distribution of the edge-to-edge gap distance after UV ozone treatment.

is located at $\sim 717 \mathrm{~nm}$ (Figure $2 \mathrm{a}) .^{31}$ The transmission electron microscopy image (inset to Figure 2a) suggests that the nanorods exhibit good size uniformity. Ten microliter Au nanorod dispersion containing $\mathrm{NaCl}$ $(0.01 \mathrm{M})$ was drop-casted on Si substrates kept in a Petri dish at room temperature $\left(\sim 21^{\circ} \mathrm{C}\right)$. After $12 \mathrm{~h}$, the vivid hexagonally vertical arrays were formed, and the edgeto-edge gap distance between the adjacent Au nanorods was found to be $7.7 \pm 0.4 \mathrm{~nm}$ (Figure $2 \mathrm{~b}$ ). The inset in Figure $2 b$ shows the edge image which clearly indicates that the array is in monolayer fashion. In order to tune the gap distance between the adjacent $\mathrm{Au}$ nanorods, the ionic strength was adjusted to change the Debye length. ${ }^{32}$ In a colloidal aqueous dispersion, the Debye length for a symmetric monovalent electrolyte is usually denoted by $\kappa^{-1}(\mathrm{~nm})=$ $0.3 /(I(M))^{1 / 2}{ }^{3}$ where $l$ is the ionic strength expressed in molar ( $\mathrm{mol} / \mathrm{L}$ ). In our experiments, $\mathrm{NaCl}$ is used as the electrolyte to adjust the ionic strength. The edge-toedge gap distance decreases as the Debye length decreases. When the Debye length is adjusted to be $1.7 \mathrm{~nm}$, the gap distance between adjacent vertical $\mathrm{Au}$ rods decreases to $6.7 \pm 0.9 \mathrm{~nm}$, which is approximately twice the length of a bilayer of the cationic surfactant $\mathrm{CTAB} .{ }^{34}$ We believe this value is the lower limit we can achieve in assembly, and the gap distance between adjacent rods is tuned to $7.7 \pm 0.4,7.9 \pm 0.9,10.2 \pm 0.7$, and $11.8 \pm 0.8 \mathrm{~nm}$ in the cases where the Debye lengths are 3.0, 4.4, 7.2, and $11.3 \mathrm{~nm}$, respectively (Figure 2c). To further decrease the gap distance, the CTAB molecules on the nanorod surface attached during synthesis were removed. The vertical Au nanorod monolayer with a $\sim 7.7 \mathrm{~nm}$ gap distance was treated by UV ozone cleaning for 20 min at room temperature. Surprisingly, the edge-to-edge gap distance decreases to $0.8 \pm 0.3 \mathrm{~nm}$, and crack patterns are formed (Figure $2 \mathrm{~d}, \mathrm{e})$. The gap distance predominantly distributes between 0.6 and $1.0 \mathrm{~nm}$ (Figure 2f). We suggest that this is because electrostatic repulsive force diminishes as the CTAB molecules are removed. The van der Waals forces further drag the nanorods closer. This leads to the crack pattern and subnanometer gap distance formation in each domain. As a result, the local electromagnetic enhancement can be dramatically improved upon UV ozone treatment according to Figure $1 \mathrm{c}$. To better understand the decrease of gap size, we have used the cathodoluminescence $(\mathrm{CL})$ system to investigate the plasmon response, ${ }^{35,36}$ which is excited by a focused $8 \mathrm{kV}$ electron beam. After the treatment by UV ozone, the CL spectra show a red shift from 537 to $575 \mathrm{~nm}$ (Figure 3), indicating the gap size decrease, which is consistent with a previous theoretical work. ${ }^{37}$ Meanwhile, the $\mathrm{CL}$ intensity increases sharply, which indicates that the plasmon coupling is enhanced, further verifying the decrease of gap size.

Mechanism of Self-Assembly of Au Nanorods. We found that highly organized vertical monolayer arrays of $\mathrm{Au}$ nanorods can be obtained on a variety of substrates, ranging from indium tin oxide (ITO)-covered glass, GaN light-emitting diode (LED) devices, glass substrates, and even flexible PEN films (Supporting Information Figure S1), which is thus of high interest for a wide range of potential applications, such as the control on the directionality and polarization of LEDs, the light enhancer in flexible electronic and photonic devices. ${ }^{38}$ The results indicate that the formation of the vertically aligned monolayer is independent of substrates. Therefore, we propose that the assembly follows the following mechanism (Figure $4 a-c)$ ): First, Au nanorods in the aqueous dispersion form the hexagonal initial 
nucleus in a side-to-side model, and then the free $\mathrm{Au}$ nanorods assemble around the nucleus, leading to the growth of the hexagonal monolayer arrays (Supporting Information Figure S2). Finally, the monolayer arrays precipitate to stand up on the substrate due to the gravity and van der Waal's interactions to the host substrates. The fact that a few monolayer arrays lie down on the substrate horizontally after formation further proves our mechanism (Figure 4d). However, the vertical monolayer array predominates because it has a larger contact area with respect to the substrate and is subjected to the minimization of surface energy. During the self-assembly process, the van der Waals force and attractive depletion force induce adjacent $\mathrm{Au}$
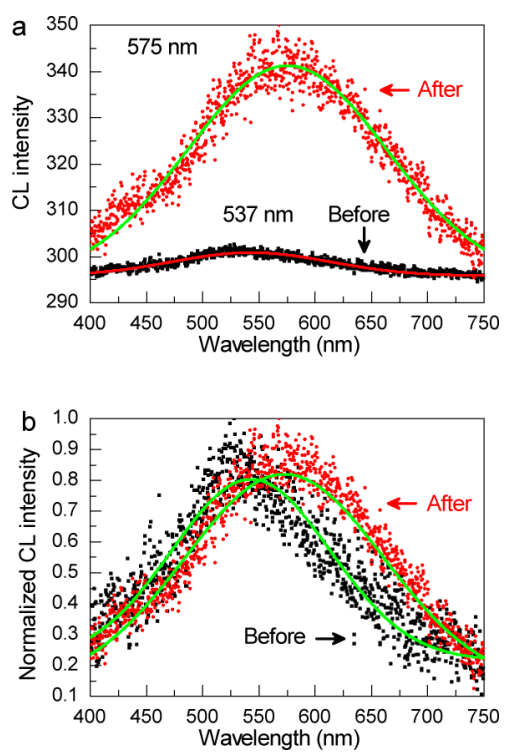

Figure 3. (a) CL spectra of the vertically aligned Au nanorod monolayer before and after treatment by UV ozone. (b) Normalized CL spectra from (a), which clearly shows the red shift after UV ozone treatment. nanorods to approach each other. Electrostatic repulsive force is responsible for stabilizing the Au nanorods within a certain distance and prevents them from random aggregation. The synergy between the attractive and repulsive force induces Au nanorods to highly ordered phases. To calculate the electrostatic energy between two similar parallel rods, the Derjaguin's approximation is used, which assumes that the parallel rods are considered to be formed by the contributions of many slices of parallel thin plates. The electrostatic interaction energy per unit area between two similar parallel plates, $E_{\mathrm{pl}}$, can be written as ${ }^{39}$

$$
E_{\mathrm{pl}}=\varepsilon \varepsilon_{0} \kappa \varphi^{2}\left[1-\tanh \left(\kappa \frac{h-2 t_{\mathrm{CTAB}}}{2}\right)\right]
$$

where $\varepsilon$ and $\varepsilon_{0}$ are the relative electric permittivity of the electrolyte solution and the electric permittivity of a vacuum, respectively; $\kappa$ is the inverse Debye length; $\varphi$ is the zeta-potential of Au rods; $h$ is the edge-to-edge gap distance between plates; $t_{C T A B}$ is the thickness of the CTAB bilayer on the surface of the plate, about $3.2 \mathrm{~nm} \cdot{ }^{39,40}$ With the help of Derjaguin's approximation, the electrostatic energy between two similar parallel rods, $E_{\text {ele, }}$ is given by ${ }^{41}$

$$
\begin{aligned}
E_{\mathrm{ele}} & =I \int_{-\infty}^{\infty} E_{\mathrm{pl}} \mathrm{d} x \\
& =2 I \sqrt{\pi \kappa r} \varepsilon \varepsilon_{0} \varphi^{2} L i_{1 / 2}\left(-e^{-\kappa\left(h-2 t_{(\top \mathrm{AB})}\right)}\right.
\end{aligned}
$$

where $l$ is the length of the Au rod, $r$ is the radius of the Au rod; $k r \gg 1$ and $\left(h-2 t_{\text {CTAB }}\right) \ll r . L i_{s}(x)$ is the polylogarithm function, defined by $L i_{s}(x)=\sum_{k=1}^{\infty}\left(x^{k} / k^{s}\right)$. The van der Waals $E_{\mathrm{vdw}}$, depletion $E_{\mathrm{dep}}$, and electrostatic energy as a function of gap distance of $h$ were calculated (Supporting Information) in the case of the Debye length of $\sim 3.0 \mathrm{~nm}$ (Figure 4e). ${ }^{26,42}$ The depletion interaction is much smaller than van der Waals and
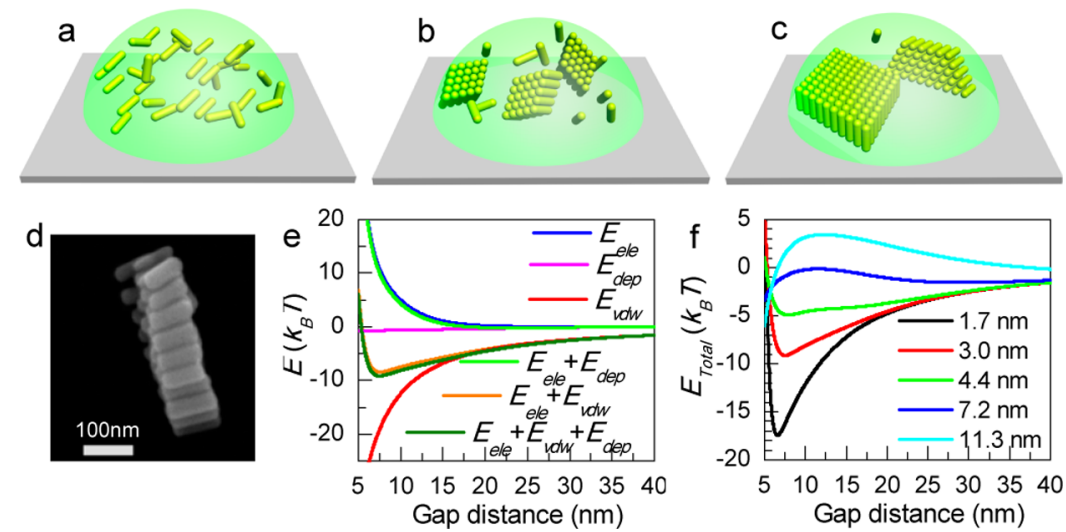

Figure 4. Schematic cartoons of the evolution of vertical Au nanorod self-assembly arrays in different stages: (a) the initial dispersion of the Au nanorods in the $\mathrm{NaCl}$ aqueous solution, (b) the nucleation and growth process of the self-assemblies, (c) the sedimentation and the final formation of the Au nanorod vertical arrays. (d) SEM image of a monolayer Au nanorod array lying down on the substrate horizontally. (e,f) Theoretical calculation results for the self-assemblies. (e) Interaction free energy as a function of the gap distance between two Au nanorods in the case that the Debye length $k^{-1}$ is $3 \mathrm{~nm}$. (f) Total interaction energy $E_{\text {Total }}$ as a function of gap distance between two parallel Au nanorods in the case that $k^{-1}$ is $11.3,7.2,4.4,3$, and $1.7 \mathrm{~nm}$. 
electrostatic interaction. If only the electrostatic and depletion interactions are considered, the resultant force is still repulsive, but the synergy of electrostatic and van der Waals interactions results in the energy minimized at $7.5 \mathrm{~nm}$, which is in good agreement with our experimental data $(\sim 7.7 \mathrm{~nm})$. Therefore, the electrostatic and van der Waals interactions are predominant on the formation of vertical arrays. The total interaction energy, defined by $E_{\text {Total }}=E_{\text {ele }}+E_{\text {dep }}+$ $E_{\mathrm{vdw}}$, as a function of gap distance between two parallel similar Au nanorods is shown in Figure $4 \mathrm{f}$. The free energy is minimized at $6.6,7.5$, and $7.8 \mathrm{~nm}$ when the Debye length is $1.7,3.0$, and $4.4 \mathrm{~nm}$, respectively, which is in good agreement with the experimental data, and the minimum in the interaction energy becomes deeper and the gap distance between two Au nanorods decreases as Debye length decreases. However, when the Debye length is 7.2 and $11.3 \mathrm{~nm}$, eq 2 is no longer applicable because $\kappa r$ values are 2.4 and 1.5 , respectively, which are close to 1 . Therefore, the free energy is maximized at 10.6 and $11.8 \mathrm{~nm}$, suggesting that it is not possible to assemble nanorods in a vertical monolayer array under such ionic strength.

Femtomolar Detection of Phthalates and Melamine. The unique vertical monolayer arrays are exploited for the ultrasensitive detection of food contaminants such as plasticizers and melamine. Phthalate is known as an endocrine disrupter which produces reproductive and developmental toxicity, such as miscarriage, fewer motile sperm, and external sex organ malformation in infants. ${ }^{43}$ Melamine, known as a triazine heterocyclic organic chemical material, can block and damage the renal cells, resulting in kidney malfunction and even death in infants. ${ }^{6}$ We used the highly aligned vertical monolayer of Au nanorods with a $\sim 0.8 \mathrm{~nm}$ edge-toedge gap distance as the SERS substrates and selected benzylbutylphthalate ( $\mathrm{BBP}, \mathrm{C}_{19} \mathrm{H}_{20} \mathrm{O}_{4}$ ) to investigate quantitatively the SERS signal. The Raman scattering spectroscopy was conducted with a $785 \mathrm{~nm}$ excitation. The signal interference due to CTAB molecules was eliminated by UV ozone cleaning. The characteristic peaks of CTAB molecules at $755 \mathrm{~cm}^{-1}\left(-\mathrm{C}-\mathrm{N}^{+}\right.$stretching vibration), $844 \mathrm{~cm}^{-1}\left(\mathrm{CH}_{3}\right.$ deformation), $1144 \mathrm{~cm}^{-1}$ (C-C stretching vibration), and $1266 \mathrm{~cm}^{-1}\left(\mathrm{CH}_{2}\right.$ wagging vibration) disappear compared with the Raman spectrum of $C T A B$ molecules before UV ozone (Figure $5 a$ ), which verify that $C T A B$ molecules are removed completely. When a $10 \mu \mathrm{L}$ solution of BBP ( $1 \mathrm{fM}$ ) in ethanol was drop-casted on the vertical Au rod monolayer arrays standing on $1 \times 1 \mathrm{~cm}^{2} \mathrm{Si}$ substrate, a pronounced Raman signal was observed (Figure $5 \mathrm{a}, 10 \mu \mathrm{L} 1 \mathrm{fM}$ ). As the concentration and volume of BBP solution increase, the overall spectral intensity is rather quantitatively increased, with similar spectral features (Figure 5a, from $1 \mathrm{fM}$ to $1 \mathrm{nM}$ ). The Raman peaks at 1066, 1580, and $1618 \mathrm{~cm}^{-1}$ correspond to the ring-ring stretching
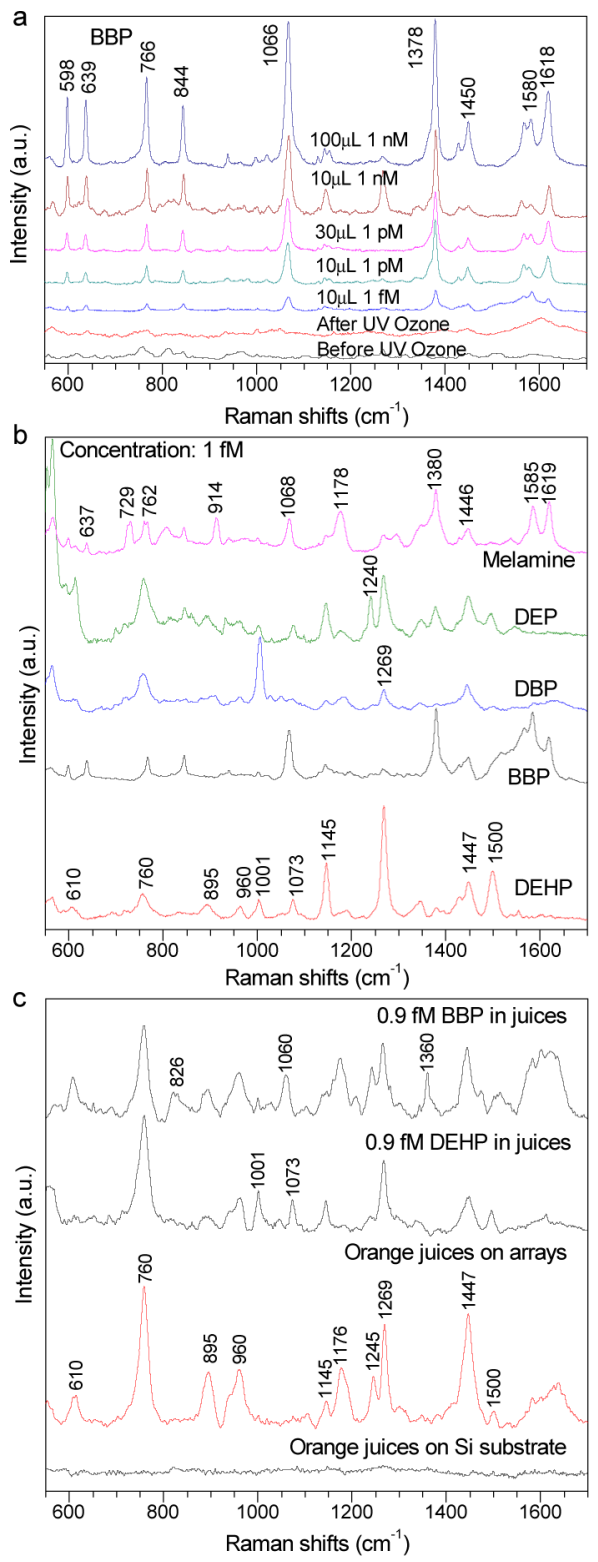

Figure 5. Femtomolar detection of plasticizer and melamine solutions. (a) SERS spectra of BBP on vertically aligned Au nanorod monolayer, from bottom to top: CTAB-coated Au nanorod arrays before and after treatment by UV ozone, $10 \mu \mathrm{L} 1 \mathrm{fM}$ BBP, $10 \mu \mathrm{L} 1 \mathrm{pM}$ BBP, $30 \mu \mathrm{L} 1 \mathrm{pM} \mathrm{BBP}, 10 \mu \mathrm{L} 1 \mathrm{nM}$ BBP, $100 \mu \mathrm{L} 1 \mathrm{nM}$ BBP. (b) SERS spectra of plasticizer (1 fM) from bottom to top: $20 \mu \mathrm{L} 1 \mathrm{fM}$ DEHP, the zoomed-in view of $10 \mu \mathrm{L} 1 \mathrm{fM}$ BBP in (a), $20 \mu \mathrm{L} 1 \mathrm{fM}$ DBP, $20 \mu \mathrm{L} 1 \mathrm{fM} \mathrm{DEP}$, and $10 \mu \mathrm{L} 1 \mathrm{fM}$ melamine. (c) Detection of BBP and DEHP in orange juices. SERS spectra from bottom to top: orange juices on $\mathrm{Si}$ substrate, $10 \mu \mathrm{L}$ mixture solution of orange juice and ethanol, DEHP (0.9 fM), and BBP $(0.9 \mathrm{fM})$ in mixture solution on vertical Au nanorod monolayer.

vibration of the ortho-phenyl group, and the peaks at 598,639 , and $766 \mathrm{~cm}^{-1}$ are due to ring deformation. The peaks at 844,1378 , and $1450 \mathrm{~cm}^{-1}$ have been assigned to the aromatic $\mathrm{C}-\mathrm{H}$ twisting vibration, the $\mathrm{CH}_{3}$ symmetric deformation, and the $\mathrm{C}-\mathrm{H}$ in-plane bending of the alkyl group, respectively. ${ }^{44,45}$ On the basis of the highly sensitive SERS substrates, we can also detect melamine $\left(\mathrm{C}_{3} \mathrm{H}_{6} \mathrm{~N}_{6}\right)$ and other plasticizers at 
femtomolar levels, such as bis(2-ethylhexyl)phthalate (DEHP, $\mathrm{C}_{24} \mathrm{H}_{38} \mathrm{O}_{4}$ ), dibutyl phthalate (DBP, $\mathrm{C}_{16} \mathrm{H}_{22} \mathrm{O}_{4}$ ), and diethyl phthalate (DEP, $\mathrm{C}_{12} \mathrm{H}_{14} \mathrm{O}_{4}$ ) (Figure 5b). For melamine, the peaks at 637 and $914 \mathrm{~cm}^{-1}$ have been assigned to the ring breathing mode, involving an inplane deformation of the triazine ring. The peaks at 729 and $762 \mathrm{~cm}^{-1}$ correspond to the out-of-plane vibration of the ring. The ring stretching vibrations are at 1068 and $1078 \mathrm{~cm}^{-1} ; 1350-1450 \mathrm{~cm}^{-1}$ is assigned to a semicircle ring stretch. The quadrant ring stretching vibrations are in $1500-1600 \mathrm{~cm}^{-1}$ region. The peak at $1620 \mathrm{~cm}^{-1}$ is from $\mathrm{NH}_{2}$ deformation. ${ }^{46-48}$ Such femtomolar level sensitivity and molecule fingerprint identification suggest that the vertical $\mathrm{Au}$ nanorod arrays pave a way to direct and fast detection of food contaminants in the real world, such as food or drinks.

We demonstrate this concept by designing an experiment to detect trace amounts of BBP and DEHP $(0.9 \mathrm{fM})$ in orange juice. Ten microliters of orange juice sample was extracted from a bottle of a commercial brand orange juice from a grocery store in Singapore. We then mixed the orange juice sample with a phthalate plasticizer (BBP or DEHP) ethanol solution (90 $\mu \mathrm{L}, 1 \mathrm{fM}$ ), so the actual BBP or DEHP concentration was $\sim 0.9 \mathrm{fM}$. Besides water, pure orange juice mainly contains Vitamin $C$ and carotene. Direct Raman analysis of pure orange juice on Si substrates did not offer any detectable Raman signals (Figure $5 c$ ), but the SERS spectrum of orange juice taken on vertical Au nanorod arrays (Figure $5 \mathrm{C}$ ) showed the fingerprint features of Vitamin $\mathrm{C}$ and carotene at 610 ( $\mathrm{C}-\mathrm{C}$ ring stretching), 760 (OH out-of-plane deformation), 895 (C-C ring stretching), $960(\mathrm{C}-\mathrm{H}$ and $\mathrm{O}-\mathrm{H}$ in-plane bending), 1145 (C-O-C stretching), 1176 (methyl rock), 1245 (C-O-H bending), 1269 (C-O-C stretching), 1447 ( $\mathrm{C}-\mathrm{H}$ scissoring), and $1500\left(\mathrm{C}=\mathrm{C}\right.$ stretching $\mathrm{cm}^{-1}$, with the corresponding vibrational modes identified in the brackets. ${ }^{49,50}$ After addition of DEHP in orange juice at $0.9 \mathrm{fM}$, the Raman peaks of DEHP at 1001 and $1073 \mathrm{~cm}^{-1}$, which were assigned to the ring-ring stretching mode, were detected in comparison to the SERS spectrum of the orange juice (Figure $5 c$ ). For the BBP-contaminated orange juice, the Raman peaks of $B B P$ at 826,1060 , and $1360 \mathrm{~cm}^{-1}$ were clearly identified
(Figure 5c), although a few wavenumbers shift to lower energy is observed in comparison to BBP molecules in ethanol (Figure 5a), which is due to the intermolecular interactions between BBP molecules and Vitamin C in juices by the hydrogen bond and $\pi$-electrons. ${ }^{51}$ It is important to note that BBP and DEHP can be distinguished unambiguously, although they share some similar Raman fingerprints. Our results indicate that the plasticizer contaminations in the actual orange juice sample can be detected at femtomolar level, suggesting the great potential of a simple nanorod monolayer for practical applications in food safety and environmental screening.

\section{CONCLUSION}

In summary, we have demonstrated an evaporationinduced self-assembly of CTAB-coated Au nanorods to highly organized vertical monolayer arrays, which can be reproducibly prepared on arbitrary substrates, such as silicon, glass, GaN LEDs, and flexible PEN polymer. The edge-to-edge gap distance between adjacent $\mathrm{Au}$ nanorods can be tuned between 11.8 and $6.7 \mathrm{~nm}$ by adjusting the ionic strength. The synergy between the electrostatic repulsive force and the van der Waals attractive force keeps the Au nanorod arrays at equilibrium status. The decrease of electrostatic force will induce Au nanorods to approach each other in ambient environment, which can result in smaller edge-toedge gap. Our experimental data show that the gap can decrease to the sub-nanometer regime by removing the CTAB molecules absorbed on Au nanorods by UV ozone treatment. FDTD calculation indeed suggests that the vertical monolayer arrays exhibit a strong and uniform electric field enhancement in the gap on the order of $10^{6}$ at the sub-nanometer gap distance, which results in the exceptional femtomolar detection of melamine and a variety of plasticizers in SERS with the molecular fingerprint clearly resolved. More importantly, the food contaminations, such as plasticizers, can be identified unambiguously using our devices at femtomolar level in orange juice, which is $\sim 7$ orders of magnitude lower than the safety limit allowed by the FDA. Therefore, the vertically aligned Au nanorod monolayer can be used as an ideal SERS-active substrate for organic chemical molecules, virus, protein, and DNA detection, even at the single-molecule level.

\section{METHODS}

Synthesis of CTAB-Coated Au Nanorods. Au nanorods were prepared using a seeded growth method with hexadecyltrimethylammonium bromide (CTAB) as stabilizing agents. ${ }^{31}$ The seed solution was prepared by mixing the aqueous solution of fresh ice-cold $\mathrm{NaBH}_{4}(0.6 \mathrm{~mL}, 0.1 \mathrm{M}), \mathrm{HAuCl}_{4}(0.25 \mathrm{~mL} 0.01 \mathrm{M})$, and CTAB (9.75 mL 0.1 M). After rapid inversion for $2 \mathrm{~min}$, the seed solution was allowed to stand for $2 \mathrm{~h}$ at $28{ }^{\circ} \mathrm{C}$. The growth solution was made by sequentially adding the aqueous solution of $\mathrm{AgNO}_{3}(0.01 \mathrm{M}, 0.4 \mathrm{~mL}), \mathrm{HAuCl}_{4}(0.01 \mathrm{M}, 2 \mathrm{~mL})$, ascorbic acid $(0.1 \mathrm{M}, 0.32 \mathrm{~mL})$, and $\mathrm{HCl}(1.0 \mathrm{M}, 0.8 \mathrm{~mL})$ into $\mathrm{CTAB}$ aqueous solution $(0.1 \mathrm{M}, 40 \mathrm{~mL})$. Then, the seed solution $(10 \mu \mathrm{L})$ was added into the growth solution. After a gentle inversion for $10 \mathrm{~s}$, the mixture solution was left undisturbed for $16 \mathrm{~h}$. Finally, Au nanorods were obtained by centrifugation and redispersed in CTAB aqueous solution $(0.1 \mathrm{M}, 40 \mathrm{~mL})$.

Evaporation-Induced Self-Assembly of CTAB-Coated Au Nanorods to Vertically Aligned Monolayer. Three milliliters of prepared Au nanorods was centrifugated at the speed of $6000 \mathrm{rpm}$ for $10 \mathrm{~min}$, and the precipitates were redispersed in CTAB solution ( $2 \mathrm{~mL}, 2.5 \mathrm{mM}$ ) containing $\mathrm{NaCl}$ whose concentration was $0,0.001,0.004,0.01$, and $0.03 \mathrm{M}$. Then, the $10 \mu \mathrm{L}$ dispersion of 
CTAB-coated Au nanorods was dropped on arbitrary substrates, such as $\mathrm{Si}$, glass, ITO glass, GaN LED devices, or flexible PEN polymer, which were cleaned with acetone and isopropyl alcohol. The samples were kept in Petri dish with cover at $\sim 21{ }^{\circ} \mathrm{C}$ for $12 \mathrm{~h}$. The humidity was $\sim 70 \%$. The vertically aligned monolayer of Au nanorods was formed.

SERS and Cathodoluminescence Characterization. Raman scattering spectroscopy was conducted on the Au nanorod arrays using a micro-Raman spectrometer (Horiba-JY T64,000) excited with a solid-state laser $(\lambda=785 \mathrm{~nm})$ in the backscattering configuration. The backscattered signal was collected through a $100 \times$ objective and dispersed by a $1800 \mathrm{~g} / \mathrm{mm}$ grating, and the laser power on the sample surface was measured to about $1.2 \mathrm{~mW}$. The typical integration time is $300 \mathrm{~s}$. Quantitative cathodoluminescence was carried out using an Attolight Rosa 4634 microscope, which tightly integrates a high-speed achromatic reflective lens (N.A. 0.72 ) within the objective lens of a field emission gun scanning electron microscope (FEG-SEM). Cathodoluminescence was spectrally resolved with a Czerny-Turner spectrometer $(320 \mathrm{~mm}$ focal length, 150 grooves $/ \mathrm{mm}$ grating) and measured with an Andor Newton EM-CCD. Electron beam energies of $8 \mathrm{kV}$ were used to excite the samples. The beam dwell time was set to $1 \mathrm{~s}$

Detection of Plasticizers and Melamine. To eliminate the signal interference of CTAB molecules absorbed on the surface of $A u$ nanorods during the synthesis process, the vertical Au nanorod monolayer arrays were treated by UV ozone for 20 min without heating. The plasticizer ethanol solution and melamine methanol solution, such as BBP, DBP, DEP, and DEHP, were prepared with different concentration ( $1 \mathrm{fM}, 1 \mathrm{pM}, 1 \mathrm{nM})$. For BBP detection, $10 \mu \mathrm{L}$ of $\mathrm{BBP}$ ethanol solution (1 $\mathrm{fM}$ ) was dropped on vertical Au nanorod arrays on the $1 \times 1 \mathrm{~cm}^{2}$ Si substrate, which spread quickly. After $1 \mathrm{~h}$, ethanol was totally evaporated and SERS signals were recorded. For other plasticizers and melamine, the procedure was the same.

Plasticizer (BBP and DEHP) Detection in Orange Juice. Ten microliters of orange juice bought from market was mixed with $90 \mu \mathrm{L}$ of ethanol or plasticizer ethanol solution (BBP or DEHP, $1 \mathrm{fM}$ ). Ten microliter mixture solution was drop-casted on vertical $A u$ nanorod arrays on the $1 \times 1 \mathrm{~cm}^{2}$ Si substrate. After ethanol totally evaporated, the SERS signals were recorded.

Conflict of Interest: The authors declare no competing financial interest.

Acknowledgment. Q.X. gratefully thanks the strong support from Singapore National Research Foundation through a fellowship grant (NRF-RF2009-06) and a Competitive Research Program (NRF-CRP-6-2010-2), and Singapore Ministry of Education via two Tier2 grants (MOE2011-T2-2-051 and MOE2011-T22-085).

Supporting Information Available: SEM image of vertically aligned Au nanorod monolayer on other substrates and the nucleation and growth process of the self-assemblies, the calculation of van der Waals potential and depletion potential. This material is available free of charge via the Internet at http://pubs.acs.org.

\section{REFERENCES AND NOTES}

1. http://en.wikipedia.org/wiki/2008_Chinese_milk_scandal.

2. http://en.wikipedia.org/wiki/2011_Taiwan_food_scandal.

3. http://www.bbc.co.uk/news/world-europe-12120321.

4. Ehling, S.; Tefera, S.; Ho, I. P. High-Performance Liquid Chromatographic Method for the Simultaneous Detection of the Adulteration of Cereal Flours with Melamine and Related Triazine By-products Ammeline, Ammelide, and Cyanuric Acid. Food Addit. Contam. 2007, 24, 1319-1325.

5. Huang, G. M.; Zheng, O. Y.; Cooks, R. G. High-Throughput Trace Melamine Analysis in Complex Mixtures. Chem. Commun. 2009, 556-558.

6. Ai, K. L.; Liu, Y. L.; Lu, L. H. Hydrogen-Bonding RecognitionInduced Color Change of Gold Nanoparticles for Visual Detection of Melamine in Raw Milk and Infant Formula. J. Am. Chem. Soc. 2009, 131, 9496-9497.
7. Cao, Q. A.; Zhao, H.; He, Y. J.; Li, X. J.; Zeng, L. X.; Ding, N.; Wang, J. A.; Yang, J.; Wang, G. W. Hydrogen-Bonding-Induced Colorimetric Detection of Melamine by NonaggregationBased Au-NPs as a Probe. Biosens. Bioelectron. 2010, 25, 2680-2685.

8. Kennedy, S. Epidemiology: Why Can't We Test Our Way to Absolute Food Safety? Science 2008, 322, 1641-1643.

9. Nie, S. M.; Emery, S. R. Probing Single Molecules and Single Nanoparticles by Surface-Enhanced Raman Scattering. Science 1997, 275, 1102-1106.

10. Yonzon, C. R.; Haynes, C. L.; Zhang, X. Y.; Walsh, J. T.; Van Duyne, R. P. A Glucose Biosensor Based on SurfaceEnhanced Raman Scattering: Improved Partition Layer, Temporal Stability, Reversibility, and Resistance to Serum Protein Interference. Anal. Chem. 2004, 76, 78-85.

11. Peng, B.; Zhang, Q.; Liu, X. F.; Ji, Y.; Demir, H. V.; Huan, C. H. A.; Sum, T. C.; Xiong, Q. H. Fluorophore-Doped Core-Multishell Spherical Plasmonic Nanocavities: Resonant Energytransfer toward a Loss Compensation. ACS Nano 2012, 6, 6250-6259.

12. Li, J. F.; Huang, Y. F.; Ding, Y.; Yang, Z. L.; Li, S. B.; Zhou, X. S.; Fan, F. R.; Zhang, W.; Zhou, Z. Y.; Wu, D. Y.; et al. ShellIsolated Nanoparticle-Enhanced Raman Spectroscopy. Nature 2010, 464, 392-395.

13. Park, W. H.; Kim, Z. H. Charge Transfer Enhancement in the SERS of a Single Molecule. Nano Lett. 2010, 10, 4040-4048.

14. Jackson, J. B.; Halas, N. J. Surface-Enhanced Raman Scattering on Tunable Plasmonic Nanoparticle Substrates. Proc. Natl. Acad. Sci. U.S.A. 2004, 101, 17930-17935.

15. Anker, J. N.; Hall, W. P.; Lyandres, O.; Shah, N. C.; Zhao, J.; Van Duyne, R. P. Biosensing with Plasmonic Nanosensors. Nat. Mater. 2008, 7, 442-453.

16. Lim, D. K.; Jeon, K. S.; Hwang, J. H.; Kim, H.; Kwon, S.; Suh, Y. D.; Nam, J. M. Highly Uniform and Reproducible Surface-Enhanced Raman Scattering from DNA-Tailorable Nanoparticles with 1-nm Interior Gap. Nat. Nanotechnol. 2011, 6, 452-460.

17. Alvarez-Puebla, R. A.; Agarwal, A.; Manna, P.; Khanal, B. P.; Aldeanueva-Potel, P.; Carbo-Argibay, E.; Pazos-Perez, N.; Vigderman, L.; Zubarev, E. R.; Kotov, N. A.; et al. Gold Nanorods 3d-Supercrystals as Surface Enhanced Raman Scattering Spectroscopy Substrates for the Rapid Detection of Scrambled Prions. Proc. Natl. Acad. Sci. U.S.A. 2011, 108, 8157-8161.

18. Dodson, S.; Haggui, M.; Bachelot, R.; Plain, J.; Li, S.; Xiong, Q. Optimizing Electromagnetic Hotspots in Plasmonic Bowtie Nanoantennae. J. Phys. Chem. Lett. 2013, 4, 496-501.

19. Johnson, P. B.; Christy, R. W. Optical Constants of the Noble Metals. Phys. Rev. B 1972, 6, 4370-4379.

20. Bico, J.; Thiele, U.; Quere, D. Wetting of Textured Surfaces. Colloids Surf., A 2002, 206, 41-46.

21. Peng, B.; Tan, L. F.; Chen, D.; Meng, X. W.; Tang, F. Q. Programming Surface Morphology of $\mathrm{TiO}_{2}$ Hollow Spheres and Their Superhydrophilic Films. ACS Appl. Mater. Interfaces 2012, 4, 96-101.

22. Wang, L. F.; Zhao, Y.; Wang, J. M.; Hong, X.; Zhai, J.; Jiang, L.; Wang, F. S. Ultra-Fast Spreading on Superhydrophilic Fibrous Mesh with Nanochannels. Appl. Surf. Sci. 2009, 255, 4944-4949.

23. Docket No. FDA 1993-N-0259 (Formerly Docket No. 1993n0085).

24. Ming, T.; Kou, X.; Chen, H.; Wang, T.; Tam, H.-L.; Cheah, K.-W.; Chen, J.-Y.; Wang, J. Ordered Gold Nanostructure Assemblies Formed by Droplet Evaporation. Angew. Chem., Int. Ed. 2008, 47, 9685-9690.

25. Guerrero-Martínez, A.; Pérez-Juste, J.; Carbó-Argibay, E.; Tardajos, G.; Liz-Marzán, L. M. Gemini-Surfactant-Directed Self-Assembly of Monodisperse Gold Nanorods into Standing Superlattices. Angew. Chem., Int. Ed. 2009, 48, 9484-9488.

26. Xie, Y.; Guo, S.; Ji, Y.; Guo, C.; Liu, X.; Chen, Z.; Wu, X.; Liu, Q. Self-Assembly of Gold Nanorods into Symmetric Superlattices Directed by OH-Terminated Hexa(ethylene glycol) Alkanethiol. Langmuir 2011, 27, 11394-11400.

27. Ng, K. C.; Udagedara, I. B.; Rukhlenko, I. D.; Chen, Y.; Tang, Y.; Premaratne, M.; Cheng, W. L. Free-Standing PlasmonicNanorod Super Lattice Sheets. ACS Nano 2012, 6, 925-934. 
28. Peng, B.; Chen, D.; Deng, Z. T.; Wen, T.; Meng, X. W.; Ren, X. L.; Ren, J.; Tang, F. Q. Surfactant-Free Self-Assembly of Nanocrystals into Ellipsoidal Architectures. ChemPhysChem 2010, 11, 3744-3751.

29. Peng, B.; Deng, Z. T.; Tang, F. Q.; Chen, D.; Ren, X. L.; Ren, J. Self-Healing Self-Assembly of Aspect-Ratio-Tunable Chloroplast-Shaped Architectures. Cryst. Growth Des. 2009, 9, 4745-4751.

30. Thai, T.; Zheng, Y. H.; Ng, S. H.; Mudie, S.; Altissimo, M.; Bach, U. Self-Assembly of Vertically Aligned Gold Nanorod Arrays on Patterned Substrates. Angew. Chem., Int. Ed. 2012, 51, 8732-8735.

31. Ming, T.; Zhao, L.; Yang, Z.; Chen, H.; Sun, L.; Wang, J.; Yan, C. Strong Polarization Dependence of Plasmon-Enhanced Fluorescence on Single Gold Nanorods. Nano Lett. 2009, 9, 3896-3903.

32. Moon, G. D.; Lee, T. I.; Kim, B.; Chae, G.; Kim, J.; Kim, S.; Myoung, J. M.; Jeong, U. Assembled Monolayers of Hydrophilic Particles on Water Surfaces. ACS Nano 2011, 5, 8600-8612.

33. Israelachvili, J. Intermolecular and Surface Forces; Academic Press Inc.: New York, 1985, ISBN: 0-12-375181375180.

34. Sau, T. K.; Murphy, C. J. Self-Assembly Patterns Formed upon Solvent Evaporation of Aqueous Cetyltrimethylammonium Bromide-Coated Gold Nanoparticles of Various Shapes. Langmuir 2005, 21, 2923-2929.

35. Coenen, T.; Vesseur, E. J. R.; Polman, A.; Koenderink, A. F. Directional Emission from Plasmonic Yagi-Uda Antennas Probed by Angle-Resolved Cathodoluminescence Spectroscopy. Nano Lett. 2011, 11, 3779-3784.

36. Lassiter, J. B.; Sobhani, H.; Knight, M. W.; Mielczarek, W. S.; Nordlander, P.; Halas, N. J. Designing and Deconstructing the Fano Lineshape in Plasmonic Nanoclusters. Nano Lett. 2012, 12, 1058-1062.

37. Funston, A. M.; Novo, C.; Davis, T. J.; Mulvaney, P. Plasmon Coupling of Gold Nanorods at Short Distances and in Different Geometries. Nano Lett. 2009, 9, 1651-1658.

38. Xu, X. L.; Peng, B.; Li, D. H.; Zhang, J.; Wong, L. M.; Zhang, Q.; Wang, S. J.; Xiong, Q. H. Flexible Visible-Infrared Metamaterials and Their Applications in Highly Sensitive Chemical and Biological Sensing. Nano Lett. 2011, 11, 3232-3238.

39. Young, K. L.; Jones, M. R.; Zhang, J.; Macfarlane, R. J.; Esquivel-Sirvent, R.; Nap, R. J.; Wu, J. S.; Schatz, G. C.; Lee, B.; Mirkin, C. A. Assembly of Reconfigurable One-Dimensional Colloidal Superlattices Due to a Synergy of Fundamental Nanoscale Forces. Proc. Natl. Acad. Sci. U.S.A. 2012, 109, 2240-2245.

40. Alkilany, A. M.; Frey, R. L.; Ferry, J. L.; Murphy, C. J. Gold Nanorods as Nanoadmicelles: 1-Naphthol Partitioning into a Nanorod-Bound Surfactant Bilayer. Langmuir 2008, 24, 10235-10239.

41. Ohshima, H.; Hyono, A. Electrostatic Interaction between Two Cylindrical Soft Particles. J. Colloid Interface Sci. 2009, 333, 202-208.

42. Bishop, K. J. M.; Wilmer, C. E.; Soh, S.; Grzybowski, B. A. Nanoscale Forces and Their Uses in Self-Assembly. Small 2009, 5, 1600-1630.

43. Lyche, J. L.; Gutleb, A. C.; Bergman, A.; Eriksen, G. S.; Murk, A. J.; Ropstad, E.; Saunders, M.; Skaare, J. U. Reproductive and Developmental Toxicity of Phthalates. J. Toxicol. Environ. Health, Part B 2009, 12, 225-249.

44. Norbygaard, T.; Berg, R. W. Analysis of Phthalate Ester Content in Poly(vinyl chloride) Plastics by Means of Fourier Transform Raman Spectroscopy. Appl. Spectrosc. 2004, 58, 410-413.

45. Alvarez-Puebla, R. A.; Dos Santos, D. S.; Aroca, R. F. SurfaceEnhanced Raman Scattering for Ultrasensitive Chemical Analysis of 1 and 2-Naphthalenethiols. Analyst 2004, 129, 1251-1256.

46. Larkin, P. Infrared and Raman Spectroscopy: Principles and Spectral Interpretation; Elsevier: Amsterdam, 2011, ISBN: 978-0-12-386984-5.

47. Liu, X. R.; Zinin, P. V.; Ming, L. C.; Acosta, T.; Sharma, S. K.; Misra, A. K.; Hong, S. M. Raman Spectroscopy of Melamine at High Pressures. Int. Conf. High Pressure Sci. Technol., Joint Airapt-22 and Hpcj-50 2010, 215.

48. Jurgens, B.; Irran, E.; Senker, J.; Kroll, P.; Muller, H.; Schnick, W. Melem (2,5,8-Triamino-tri-S-triazine), an Important Intermediate during Condensation of Melamine Rings to Graphitic Carbon Nitride: Synthesis, Structure Determination by X-ray Powder Diffractometry, Solid-State NMR, and Theoretical Studies. J. Am. Chem. Soc. 2003, 125, 1028810300.

49. Tschirner, N.; Schenderlein, M.; Brose, K.; Schlodder, E.; Mroginski, M. A.; Thomsen, C.; Hildebrandt, P. Resonance Raman Spectra of $\beta$-Carotene in Solution and in Photosystems Revisited: An Experimental and Theoretical Study. Phys. Chem. Chem. Phys. 2009, 11, 11471-11478.

50. Panicker, C. Y.; Varghese, H. T.; Philip, D. FT-IR, FT-Raman and SERS Spectra of Vitamin C. Spectrochim. Acta, Part A 2006, 65, 802-804.

51. Karpfen, A.; Kryachko, E. S. On Blue Shifts of C-H Stretching Modes of Dimethyl Ether in Hydrogen- and HalogenBonded Complexes. Chem. Phys. Lett. 2006, 431, 428-433. 